\title{
Overlap Between Systemic Lupus Erythematosus and Rheumatoid Arthritis: Is It Real or Just an Illusion?
}

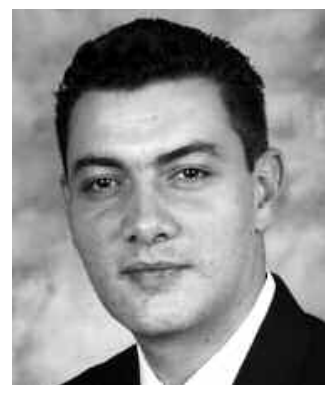

The association between rheumatoid arthritis (RA) and systemic lupus erythematosus (SLE) has been debated for many years. Based on their different genetic background and pathogenic mechanisms, it has been argued that both diseases are complex, mutually exclusive entities. However, recent knowledge is challenging this notion, suggesting that both diseases can indeed overlap.

In this issue of The Journal, Icen and colleagues explore the frequency of SLE features and their influence on mortality in an incident cohort of 603 subjects with RA followed over time $^{1}$. This study was conducted in the Rochester Epidemiology Project, a centralized medical record-linkage system that encompasses all healthcare data from residents of Rochester and Olmsted County, Minnesota, USA, since 1909. This kind of project provides an extraordinary opportunity to conduct comprehensive population-based studies over long periods. A cumulative incidence of $\geq 4$ SLE features (including arthritis) was found in $15.5 \%$ of patients after 25 years of followup, this being associated with a 2fold increase in the risk of death.

Is there an explanation for the high incidence of SLE features found in this cohort of RA patients? Information about SLE and RA association is scarce and discrepant. Cohen and colleagues reported that 11 of 309 (3.6\%) patients with SLE fulfilled classification criteria for RA, suggesting that both diseases are more frequently associated than expected by chance $^{2}$. In contrast, in a retrospective study including about 7000 new patients, Panush and colleagues identified only 6 patients with overlapping criteria for RA and SLE, with a 10 -fold lower concurrent prevalence rate $(0.09 \%)$ than that expected by chance $(1.2 \%)^{3}$.

With these data, whether RA and SLE are directly or inversely related entities remains unclear; nonetheless, the surprising finding of $15.5 \%$ of RA patients fulfilling classification criteria for SLE opens 2 options for interpretation: (1) the existing gaps in the classification systems make them not specific enough for differentiating some cases of RA from SLE, and/or (2) there is a real association between both diseases, in which case the pathogenic mechanisms underlying each of them should be revised.

Regarding classification criteria for rheumatic diseases, almost all have been performed on cross-sectional evaluations, hence they seem to neither adequately evaluate clinical and serological features collected over time nor assess the dynamic course of the corresponding disease. When the 1982 revised classification criteria for SLE were formulated, the objective was to reliably discriminate SLE patients from a heterogeneous group of inflammatory arthritides (most of them RA $)^{4}$. In spite of that, some of these criteria are also common manifestations in RA (i.e., arthritis, pleuritis, pericarditis, or proteinuria) or are related to therapy (i.e., rash, photosensitivity, oral ulcers, proteinuria, leukopenia, and antinuclear antibodies). Thus, a significant number of RA patients potentially meet SLE classification criteria. Similarly, the 1987 revised classification criteria for RA intended to improve the specificity of the former criteria, with a better discrimination between patients with well established RA versus patients with other rheumatic diseases $(20 \% \text { of them with SLE) })^{5}$. Since a number of non-RA patients also met some RA classification criteria, a warning was included: "four conditions (systemic lupus erythematosus, psoriatic arthritis, mixed connective tissue disease, and Reiter's syndrome) appear likely to have substantial numbers of patients who might fulfill the requirements of the new criteria, and caution should be observed in these circumstances".

Following this line of thought, it may be concluded that sequential concurrence of RA manifestations that are also included in the SLE classification criteria explains the high frequency of SLE features. However, it is clear that some patients with a well classified autoimmune rheumatic disease also may have features considered hallmarks of another one; for instance, a patient with established RA who presents with proliferative glomerulonephritis, psychosis, antidsDNA or anti-Sm antibodies, or other hallmarks of SLE. Do these clinical manifestations and autoantibodies reveal

See SLE features in RA and their effect on overall mortality, page 50 
the presence of a second autoimmune disorder in the same patient, or are they atypical features from a unique disease? Frequently, physicians are baffled by associations of autoimmune diseases, especially when features considered hallmarks of another disease occur; many clinicians are even reluctant to consider overlap of a second systemic autoimmune disease. As shown by Icen and colleagues, only 9 patients $(1.5 \%)$ were clinically diagnosed as having SLE, in spite of $15.5 \%$ fulfilling SLE classification criteria ${ }^{1}$. Why are rheumatologists unwilling to diagnose SLE-RA overlap? Although joint involvement is one of the most common SLE manifestations, only a minority of patients $(\sim 5 \%)$ develop deforming arthropathy. Typically it is characterized by a nonerosive arthropathy with correctable ulnar deviation of metacarpophalangeal joints, $\mathrm{Z}$ deformity of the thumb, and swan-neck deformity of fingers, resembling the Jaccoud's arthropathy first described in rheumatic fever. However, less than $1 \%$ of SLE patients develop erosive arthropathy indistinguishable from RA, an entity also known as rhupus 6,7 .

The first insights about the RA and SLE overlap come from 1960, when Toone and colleagues described the presence of LE cells (until then, considered as exclusive for SLE) in sera of 15 patients with RA, 8 of them with systemic manifestations ${ }^{8}$. Several following studies showed positive rheumatoid factor in almost all rhupus patients ${ }^{2,3,6,7}$. Also, anti-RA33 antibodies (previously considered specific for RA) have been associated with erosive arthritis in SLE' . However, both antibodies are detected in SLE patients with either erosive or nonerosive arthropathy and even in nondeforming, nonerosive arthropathy ${ }^{10}$.

Since almost all information available on rhupus has been circumstantial, doubts about its existence have been raised. If rhupus represents SLE and RA overlap, an SLE subset with intense joint expression, or a distinctive entity is a matter of debate. There is no discussion about rhupus being part of the SLE spectrum; the questioning has been whether these patients also have RA. Currently, there is evidence supporting the existence of rhupus as a true overlap syndrome. There are at least 3 biological markers that, being hallmarks of RA, are also present in rhupus, but not in SLE. First, an elevated production of the highly specific (96\%-98\%) anti-cyclic citrullinated peptide (anti-CCP) antibody has been demonstrated in RA patients ${ }^{11}$. We described that anti-CCP antibodies are present in similar frequencies and titration in rhupus and RA patients, but these are significantly higher than in SLE patients with nonerosive $\operatorname{arthritis}^{12}$; others later confirmed these results ${ }^{13-15}$. The strength of the association between anti-CCP and rhupus seems to be high, as the presence of these antibodies increases 18 to 28 times the risk of developing erosive arthritis in patients with SLE ${ }^{13,16}$.

Second, the close association between RA risk and different MHC class II molecules sharing the same amino acid sequence (arginine-alanine-alanine) in positions 72 to 74 of the $\beta$-chain is well established ${ }^{17}$. These alleles, collectively termed "shared epitope" (SE), preferentially bind peptides containing the non-standard amino acid citrulline (deiminated arginine). Dose-effect of the SE over the production of anti-CCP antibodies in RA has been reported; in addition, the association of the SE and erosive disease is thought to be an indirect effect mediated by antibodies against different citrullinated proteins ${ }^{18}$. As seen in RA, 67\% of SLE patients with major erosions have alleles of the SE, in contrast with only $22 \%$ of those with nonerosive arthritis. Moreover, the presence of 2 copies of the SE increases the risk of erosive arthritis in SLE 8 times $^{13}$. Besides RA and SLE, the association of SE with anti-CCP antibody production and erosive arthropathy has also been described in patients with psoriatic arthritis ${ }^{19}$. Hence, recent evidence supports the hypothesis that anti-CCP antibody response (and probably, the entire abnormal citrulline metabolism including the SE) plays a direct pathogenic role in the development of a severe and erosive arthritis phenotype, regardless of the underlying disease. Finally, apart from its extensive use as an inflammatory marker, C-reactive protein (CRP) is a pentraxin critically involved in the disposal of necrotic detritus and apoptotic particles. While CRP levels correlate directly with disease activity in RA, a failure in its production has been found in SLE, despite evident tissue inflammation. This underpowered acute-phase response results in diminished clearance of dying cells, and has been hypothesized as critical in the pathogenesis of SLE. Nevertheless, we have found that rhupus patients display adequate CRP response, with serum concentrations significantly higher than those found in SLE patients with nonerosive arthritis (14.5 vs $0.8 \mathrm{mg} / \mathrm{l}$, respectively; $p=0.01$ ), suggesting that CRP may be an active pathogenic agent in addition to its known utility as a serological marker for an erosive arthritis pattern among SLE patients ${ }^{16}$.

Longterm studies on incident cohorts imply some inherent biases and deficiencies (i.e., laboratory assays may not be available or may change throughout the study period; the clinical course and severity of the disease may change over time); however, these studies have the advantage of collecting valuable data about the course of a disease over time. Additionally, since the collected information generates more questions than answers, these are provocative approaches to initiate new areas of research.

Some issues can be raised from the above information. First, classification criteria for SLE and RA need to be reevaluated in light of the wealth of new knowledge. Second, autoimmune diseases are capable of having more manifestations than we are aware of, remembering that physicians, not nature, artificially impose dividing lines between autoimmune diseases. Finally, current pathogenetic evidence points to rhupus representing the comorbidity of RA and SLE, on the understanding that shared autoimmunity is "a concept for which the time has come" 20 .

Personal non-commercial use only. The Journal of Rheumatology Copyright @ 2009 . All rights reserved. 
LUIS M. AMEZCUA-GUERRA, MD,

Department of Immunology,

Instituto Nacional de Cardiología Ignacio Chávez,

LaSalle University School of Medicine,

Juan Badiano 1, Sección XVI,

Tlalpan, 14080, Mexico City, Mexico

Address reprint requests to Dr. Amezcua-Guerra.

E-mail: lmamezcuag@gmail.com

\section{ACKNOWLEDGMENT}

I acknowledge Drs. Angélica Vargas and Rafael Bojalil for their critiques of this report.

\section{REFERENCES}

1. Icen M, Nicola PJ, Maradit-Kremers H, et al. Systemic lupus erythematosus features in rheumatoid arthritis and their effect on overall mortality. J Rheumatol 2009;36:50-7.

2. Cohen MG, Webb J. Concurrence of rheumatoid arthritis and systemic lupus erythematosus: report of 11 cases. Ann Rheum Dis 1987;46:853-8.

3. Panush RS, Edwards NL, Longley S, Webster E. "Rhupus" syndrome. Arch Intern Med 1988;148:1633-6.

4. Tan EM, Cohen AS, Fries JF, et al. The 1982 revised criteria for the classification of systemic lupus erythematosus. Arthritis Rheum 1982;25:1271-7.

5. Arnett FC, Edworthy SM, Bloch DA, et al. The American Rheumatism Association 1987 revised criteria for the classification of rheumatoid arthritis. Arthritis Rheum 1988;31:315-24.

6. Alarcon-Segovia D, Abud-Mendoza C, Diaz-Jouanen E, Iglesias A, De los Reyes V, Hernández-Ortiz J. Deforming arthropathy of the hands in systemic lupus erythematosus. J Rheumatol 1988;15:65-9.

7. van Vugt RM, Derksen RH, Kater L, Bijlsma JW. Deforming arthropathy or lupus and rhupus hands in systemic lupus erythematosus. Ann Rheum Dis 1998;57:540-4.

8. Toone EC Jr, Irby R, Pierce EL. The LE cell in rheumatoid arthritis. Am J Med Sci 1960;240:599-608.

9. Richter Cohen M, Steiner G, Smolen JS, Isenberg DA. Erosive arthritis in systemic lupus erythematosus: analysis of a distinct clinical and serological subset. Br J Rheumatol 1998;37:421-4.

10. Mediwake R, Isenberg DA, Schellekens GA, van Venrooij WJ. Use of anti-citrullinated peptide and anti-RA33 antibodies in distinguishing erosive arthritis in patients with systemic lupus erythematosus and rheumatoid arthritis. Ann Rheum Dis 2001;60:67-8.

11. Molberg O, Sollid LM. A gut feeling for joint inflammation using coeliac disease to understand rheumatoid arthritis. Trends Immunol 2006;27:188-94.

12. Amezcua-Guerra LM, Springall R, Marquez-Velasco R, Gómez-García L, Vargas A, Bojalil R. Presence of antibodies against cyclic citrullinated peptides in patients with "rhupus": a cross-sectional study. Arthritis Res Ther 2006;8:R144.

13. Chan MT, Owen P, Dunphy J, et al. Associations of erosive arthritis with anti-cyclic citrullinated peptide antibodies and MHC Class II alleles in systemic lupus erythematosus. J Rheumatol 2008;35:77-83.

14. Martinez JB, Valero JS, Bautista AJ, et al. Erosive arthropathy: clinical variance in lupus erythematosus and association with anti-CCP case series and review of the literature. Clin Exp Rheumatol 2007;25:47-53.

15. Damian-Abrego GN, Cabiedes J, Cabral AR. Anti-citrullinated peptide antibodies in lupus patients with or without deforming arthropathy. Lupus 2008;17:300-4.

16. Amezcua-Guerra LM, Marquez-Velasco R, Bojalil R. Erosive arthritis in systemic lupus erythematosus is associated with high serum C-reactive protein and anti-cyclic citrullinated peptide antibodies. Inflamm Res; (in press).

17. du Montcel ST, Michou L, Petit-Teixeira E, et al. New classification of HLA-DRB1 alleles supports the shared epitope hypothesis of rheumatoid arthritis susceptibility. Arthritis Rheum 2005;52:1063-8.

18. van der Helm-van Mil AH, Verpoort KN, Breedveld FC, Huizinga TW, Toes RE, de Vries RR. The HLA-DRB1 shared epitope alleles are primarily a risk factor for anti-cyclic citrullinated peptide antibodies and are not independent risk factor for development of rheumatoid arthritis. Arthritis Rheum 2006;54:1117-21.

19. Korendowych E, Owen P, Ravindran J, Carmichael C, McHugh N. The clinical and genetic associations of anti-cyclic citrullinated peptide antibodies in psoriatic arthritis. Rheumatology Oxford 2005;44:1056-60.

20. Alarcon-Segovia D. Shared autoimmunity: a concept for which the time has come. Autoimmunity 2005;38:201-3.

J Rheumatol 2009;36:4-6; doi:10.3899/jrheum.081067 